\title{
Antike Inschriften während des Mittelalters nördlich der Alpen. Wahrnehmung und Instrumentalisierung
}

Der folgende Beitrag geht dem Fortbestand antiker Inschriften außerhalb der mediterranen Kernlandschaften des Imperium Romanum nach und zeigt an ausgewählten Beispielen Möglichkeiten ihrer Wahrnehmung und vor allem ihrer Vereinnahmung auf.

Die steinernen Überreste römischer Niederlassungen haben das Erscheinungsbild von Stadt und Land auch über ein halbes Jahrtausend nach dem Untergang des Römischen Weltreiches in seinen ehemaligen nordwestlichen Provinzen nachhaltig geprägt. ${ }^{1}$ Über ihre einstigen Dimensionen werden wir jedoch oftmals nur durch den Überlieferungszufall, nämlich Hinweise informiert, die in dem jeweiligen Quellenzeugnis gar nicht zu erwarten gewesen wären. Eine solche hier interessierende Notiz enthält etwa ein Itinerar aus der zweiten Hälfte des 13. Jahrhunderts, das Reiserouten von Valenciennes nach Avignon bzw. von Lyon nach Orléans und Paris beschreibt. ${ }^{2}$ Ihr zufolge hatte sich damals noch eine in ihren ehemaligen Ausdehnungen wahrnehmbare antike Gräberstraße in der Umgebung der an der oberen Marne gelegenen Kathedralstadt Langres, dem einstigen gallorömischen Hauptort der Lingonen, erhalten. Ihre Monumente wurden nun - wie auch andernorts - als sarazenischen, also heidnischen Ursprungs identifiziert: et ibi inveniuntur sepulcra Sarracenorum multa. Interessant ist zudem der Hinweis auf angeblich 500 dort um eine Kapelle lebende Eremiten, wobei die Zahl sicherlich viel zu hoch gegriffen sein dürfte. Womöglich wohnten einige der Einsiedler in den antiken Grabbauten bzw. in an diese angebauten Hütten. Unter den einzeln aufgeführten Monumenten ist auch das Grabmal eines rex Sarracenorum mit der Darstellung von Schlachtszenen: et turris est sculpta de bellis ibi factis. Dabei dürfte es sich um ein frührömisches Grabmal mit Kriegsszenen gehandelt haben, zu dem zahlreiche Parallelen aus den gallischen und germanischen Provinzen überliefert sind. ${ }^{3}$

Dem weiteren Wortlaut des Itinerars zufolge stand eine halbe Meile von Langres entfernt ein weiteres Monument, que vocatur Iulie. Diese Bezeichnung nahm vermutlich Bezug auf eine noch vorhandene Inschrift. Hier wäre an einen Personennamen zu denken, der das kaiserliche Gentiliz Iulius beinhaltete und somit auf eine Familie verwies, die unter Caesar oder Octavian/Augustus das römische Bürgerrecht erhalten hatte.

1 Vgl. Clemens 2003; Beck 2015.

2 Ruelens 1890, 322-325; Lot 1922; Berlioz 1998, 176; Clemens 2009, 326-328.

3 Gabelmann 1973; Andrikopoulou-Strack 1986, 95-106; Kremer 2009.

Ә Open Access. () 2019 Erik Beck, Lukas Clemens, publiziert von De Gruyter. (cc) BY-NC-ND Dieses Werk ist lizenziert unter der Creative Commons Attribution-NonCommercial-NoDerivatives 4.0 Lizenz. 
Von besonderem Interesse ist nun, dass mit Hilfe dieser Beschreibung womöglich ein Zusammenhang mit einer weiteren Quelle, dem berühmten sogenannten „Lingonentestament“, hergestellt werden kann. Dieses wurde im 10. Jahrhundert abgeschrieben und hat sich auf einem beidseitig beschriebenen Pergamentblatt im Handschriftenbestand der Universitätsbibliothek Basel erhalten. ${ }^{4}$ Der nicht ganz vollständig überlieferte, wohl in der zweiten Hälfte des 2. Jahrhunderts entstandene Text umfasst ausführliche Verfügungen eines reichen Bürgers der civitas Lingonum über die Errichtung und Unterhaltung seines Grabmals. Erwähnt wird dort auch der Enkel des Auftraggebers - Sextus Iulius Aquila, der Sohn des Sextus Iulius Aquilinus. Auch wenn wir mit dem Itinerar natürlich nicht den Beweis antreten können, dass es sich bei dem dort erwähnten Monument um das in dem Lingonentestament genannte Mausoleum handelte - zu zahlreich sind bekanntlich die Belege der Iulii ${ }^{5}$-, so gewinnt mit ihm die Vermutung gegenüber der geäußerten These einer Überlieferung des Testamentes auf einem Papyrus an Gewicht, dem Kopisten des 10. Jahrhunderts habe vielmehr die Inschrift an einem noch erhaltenen Bauwerk als Vorlage gedient.

Die so wahrscheinlich gemachte Überlieferungssituation in Langres - das Vorhandensein zahlreicher Sepulkralbauten noch im 13. Jahrhundert mit erhaltenen Inschriften, von denen ein ausführliches Testament rund drei Jahrhunderte zuvor kopiert werden konnte, lässt neben dem Umfang überdauerter Antike auch etwas über die Fähigkeiten erahnen, die an den Monumenten überlieferten Texte noch zu lesen bzw. zu verstehen, wobei die Wahrnehmung derartiger Zeugnisse natürlich mit fortschreitender Zeitdauer durch Verwitterungen und Teil- bis Totalzerstörungen erschwert oder gar unmöglich gemacht werden konnte: Von der Tendenz her ist die Eignung antike Inschriften zu lesen, zwar durch das ganze Mittelalter hindurch vorhanden, doch geht die Anzahl derjenigen, die hierzu in der Lage waren, seit dem 13. Jahrhundert spürbar zurück. So finden sich nun immer wieder Zeugnisse, in denen explizit die Unfähigkeit, einen Text zu verstehen, zugegeben wird. ${ }^{6}$

Anders sind hingegen noch die Umstände, bei denen eine hochmittelalterliche Auseinandersetzung mit antiken Texten deutlich wird. Im Fall eines Grabgedichtes ist die gelehrte Beschäftigung mit dem Text indirekt zu erschließen: 1966 wurde im Zuge von Restaurierungsarbeiten in der Kirche der Benediktinerabtei St. Eucharius-St. Matthias vor Trier ein im Jahr 1148 der heiligen Agatha und allen Heiligen Jungfrauen geweihter Blockaltar abgebrochen, der - wie sich hierbei herausstellte - nahezu vollständig aus wiederverwendetem Altmaterial errichtet worden war. Darunter befanden sich auch sechs Fragmente einer größeren, beidseitig mit Inschriften versehenen Kalksteinplatte, die sich weitgehend zusammensetzen ließ. Auf der einen Seite fand sich eine antike Grabinschrift mit einem Distichon und einem nichtmetrischen

4 Vgl. Kiessling 1863; siehe auch CIL XIII 5708; Hatt 1951, 66-69; Frézouls 1988, 312-314; Le Bohec 1991, 9-15.

5 Allein 420 Belege finden sich in den Bänden des CIL; vgl. Le Glay 1991, 57f.

6 Calabi Limentani 1970; Clemens 2003, 414-417; Esch 2005, $38 f$. 
Zusatz, die dem 3. Jahrhundert entstammt. ${ }^{7}$ Sie nennt einen grammaticus latinus, der im Text auch als magister studiorum und doctor Romani nobilis eloquii bezeichnet wird. Auf der Rückseite befindet sich hingegen ein mittelalterliches Grabgedicht auf einen monachus Wiserich der Abtei, bestehend aus vier (daktylischen) Hexametern. Eingerahmt ist der Text oben und unten von einem dreifachen Flechtband, das - auf der linken Seite noch erhalten - jeweils in Tierfratzen endet. Ein Mönch des Klosters namens Wiserich ist für den Beginn des 11. Jahrhunderts überliefert. Die Buchstabenformen des mittelalterlichen Textes sind offenkundig an denen der antiken Grabinschrift orientiert. Erhaltene antike, aber auch früh- oder hochmittelalterliche Grabgedichte finden sich innerhalb des Trierer Inschriftenkorpus nur extrem selten. Zwei derartige, noch dazu in einem erheblichen zeitlichen Abstand zueinander verfasste Texte auf einer Platte sind daher sicherlich kein Zufall. Sie lassen vielmehr an eine mittelalterliche Auseinandersetzung mit der römischen Inschrift, ja gewissermaßen an einen gelehrten Wettstreit klassisch geschulter Kreise innerhalb des vor Trier gelegenen Benediktinerklosters mit den antiken Grammatikern denken.

Andernorts wurden mit Hilfe antiker Inschriften Traditionen begründet: In Köln führte die Auffindung einer spätrömischen Bauinschrift zur kritischen Auseinandersetzung mit einer liebgewonnenen Lokaltradition über die Gründung des antiken Kastells von Deutz, in dessen noch bis in das Hochmittelalter hinein weitgehend erhaltenen Befestigungsmauern zu Beginn des 11. Jahrhunderts ein gleichnamiges Kloster errichtet worden war. Den Wortlaut der Inschrift teilte dessen berühmter Abt Rupert in einer um 1120 verfassten, heute verlorenen Vita sancti Heriberti mit. Der Text ist im 16. Jahrhundert kopiert worden und bildet die Grundlage für die Edition im CIL XIII (Abb. 1). ${ }^{8}$

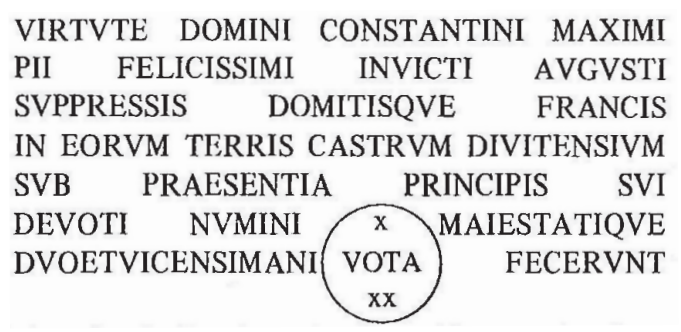

Abb. 1: Rekonstruktion von CIL XIII 8502 nach Grünewald 1989 mit dortiger Übersetzung:

Nachdem durch die virtus des Herrn Konstantin, des ranghöchsten, frommen, unbesiegbaren Augustus, die Franken niedergedrückt und gezähmt worden sind, haben in deren Gebiet Angehörige der 22. Legion, in Anwesenheit ihres Herrschers, ergeben (seinem) numen und (seiner) maiestas, das Deutzer Kastell errichtet (und ihrem Kaiser) Wünsche für weitere zehn Regierungsjahre dargebracht.

7 Cüppers/Binsfeld 1972; Die Deutschen Inschriften 2006, Bd. 70, 131-133, Abb. 44.

8 Grünewald 1989. Der Text findet sich bei Laurentius Surius 1571, 246. 
In seinem 1128 über einen verheerenden Klosterbrand abgefassten Werk De incendio berichtet Rupert dann von mehreren vor Ort zirkulierenden Meinungen: Nach der einen sei das Kastell ein Werk Julius Caesars, während die andere von einer Errichtung unter Kaiser Konstantin, nachdem dieser die Franken besiegt habe, ausgehe. Letztere Ansicht werde durch die Inschrift auf einer Steintafel gestützt, die man vor nicht allzu langer Zeit - in Teile zerbrochen - zwischen Mauerresten gefunden habe, deren Fragmente sich aber zusammenfügen ließen. ${ }^{9}$ Anschließend folgt der indirekt mitgeteilte Inhalt dieser in der Forschung heute allgemein als unbedenklich akzeptierten Bauinschrift des um 315 fertiggestellten Kastells mit dem Hinweis auf einen kurz zuvor erfolgte Frankensieg. Den Textinhalt bringt Rupert mit der bei Eutropius überlieferten Nachricht in Verbindung, man habe die Könige der unterlegenen Germanen den wilden Tieren vorgeworfen, um dann noch einmal auf die Datierungsfrage einzugehen: Weil nämlich die Taten Caesars in Gallien als sehr berühmt erachtet werden, ist die Ansicht allgemein verbreitet, wenn auch durch keine schriftlichen Zeugnisse bestärkt, dass jener der Gründer dieses Kastells sei. ${ }^{10}$ Rupert stellt folglich der lokalen Tradition die epigraphische Quelle entgegen und bringt diese mit der historiographischen Überlieferung in Verbindung, eine durchaus modern anmutende wissenschaftliche Leistung. Im Übrigen dürfte Rupert die kurz zuvor aufgefundene Inschrift nicht ungelegen gekommen sein. So musste er sein durch den Brand weitgehend zerstörtes Deutzer Kloster mit großem Aufwand wiederherstellen. In seine Restitutionspolitik passte es daher sicher sehr gut, dass sich die Tradition des Ortes mit Hilfe einer antiken Inschrift auf den ersten christlichen Kaiser zurückführen ließ.

Im Rahmen staufischer Herrschaftslegitimation spielte ein antikes Grabmonument mit erhaltener Inschrift eine offenbar nicht unerhebliche Rolle. So leitete Otto von Freising die von ihm als „Waiblinger“ bezeichneten Salier und Staufer von den Merowingern her. Wichtigstes Zeugnis für diese Geschichtskonstruktion war ein in Beinstein bei Waiblingen, also in Nähe der staufischen Stammburg sichtbarer römischer Sepulkralbau. Diesen erwähnt Burchard von Ursberg in seinem von 1126 bis 1225 geführten Chronikon:

Apparet autem usque in presens titulus monumenti iuxta prefatam villam (Waiblingen) in modum turris, miro opere de quadris et sculptis lapidibus constructus, quod vulgus Baienstein (Beinstein) denominat, in quo sculptum litteris reperitur, quod Clodius hoc fecerit uxori suae. ${ }^{11}$

9 Hanc opinionem firmiorem esse asserit titulus non multos ante annos inventus in tabula lapidea inter fragmenta murorum, et ispsa in partes divisa, ita tamen ut partes ipsae ad invicem coniugi possunt; Grundmann 1966, 449; Gechter 1989, 405 (mit Übersetzung).

10 Sed quia Iulii Cesaris res gestae in Galliis famosiores habentur, inde hec opinio magis vulgata est, licet nullis facta scriptorum monumentis, quod ille huius quoque castri conditor extiterit; Grundmann 1966, 449; Gechter 1991.

11 MGH SS 13, 338; siehe hierzu Althoff 1988, der - 422f. - in Clodius einen Offizier der römischen Limestruppen vermutet. Vgl. auch Schmid 1976, 68f.; Reuter 1992, 21; Lorenz 2000, 82. 
Der somit nur indirekt mitgeteilte Wortlaut der Inschrift nannte offenbar die Verstorbene und ihren Gatten, der das Grabmal in Auftrag gegeben hatte. Derartige Bestandteile gehörten zum festen Formular antiker Grabinschriften, so dass wir ein solches im Hochmittelalter noch weitgehend erhaltenes Bauwerk für Beinstein zwingend annehmen dürfen. ${ }^{12}$ Zumindest größere Teile der Grabinschrift sind offenbar richtig verstanden worden. Über den Namen Clodius ließ sich eine Verbindung zu den Merowingern und mit deren historiographischen Tradition dann sogar bis zurück zu den trojanischen Helden konstruieren.

Inschriften bzw. Teile von epigraphischen Überresten konnten als Spolien verbaut das Alter des eigenen Ortes visualisieren. So befand sich in einer Seitenkapelle der im 10. Jahrhundert unter Bischof Konrad (934-975) errichteten Mauritiusrotunde in Konstanz eine dort sichtbare, fragmentarische römische Inschrift (Abb. 2). ${ }^{13}$ Dem in wesentlichen Teilen zu erschließenden Inhalt des Textes zufolge handelt es sich hierbei um die Bauinschrift der Kastellmauer von Vitudurum aus dem Jahr 294. Folglich ist die Steinplatte aus dem rund 35 Kilometer entfernten Oberwinterthur, wo die Konstanzer Bischöfe seit dem Frühmittelalter über ausgedehnten Grundbesitz verfügten, vermutlich im Zuge des Baus der Mauritiusrotunde im 10. Jahrhundert auf Initiative des Bauherrn, Bischof Konrad, nach Konstanz verbracht worden. ${ }^{14}$

Die rechte Seite des Textes ist nachantik zu Teilen abgearbeitet worden, so dass der Name des in dem Text genannten Unterkaisers Constantius nun auf der Platte zentriert erscheint. ${ }^{15}$ Die Inschrift diente offenbar - wie Helmut Maurer herausgearbeitet hat - zur Demonstration der eigenen bischöflichen Tradition und sollte die Vorrangstellung des innerhalb des spätrömischen Kastells in Konstanz gelegenen Bischofssitzes gegenüber den benachbarten Reichsabteien Reichenau und St. Gallen begründen. ${ }^{16}$ Die Ortsbezeichnung Constantia leitet sich ja tatsächlich von einem spätantiken Herrscher gleichen Namens ab, vermutlich aber von Constantius II. (337-361), der im Bereich der späteren mittelalterlichen Stadt ein Kastell errichten ließ, dessen Befestigung in jüngerer Zeit auch archäologisch nachgewiesen wurde. ${ }^{17} \mathrm{Im}$ 15. Jahrhundert konnte die Inschriftenplatte nicht mehr gelesen werden. Sie wurde jedoch, wie aus einem Bericht des italienischen Humanisten Leonardo Bruni aus Arezzo sowie aus der Schedelschen Weltchronik zu erfahren ist, weiter als Gegenstand von außerge-

12 Krause 1981, 70f.

13 CIL XIII 5249. Die Inschrift befindet sich seit 1967 im Rathaus von Oberwinterthur: Drack 1968/69, bes. 159f. Zur Inschrift vgl. Maurer 1989, 70-72 und nun vor allem Maurer 2014, 200f. sowie Beck 2009, bes. 130-134. Vgl. künftig die Druckfassung der Dissertation Beck 2015, 263-265 (Abgabefassung).

14 Vgl. hierzu Clemens 2003, 409; Beck 2014, 350f.; Heiligmann 2014, 79; Maurer 2014, 200-202; Beck 2009, 130-134. Zur Mauritiusrotunde und ihrer architekturgeschichtlichen Bedeutung und zum Grab Konrads vgl. Erdmann/Zettler 1977, zur Mauritiusrotunde bes. 31-76.

15 Vgl. Maurer 1973, 80; Clemens 2003, 409; Maurer 2014, 200.

16 Maurer 2014, 200; Maurer 1973, 80.

17 Zuletzt vgl. hierzu Heiligmann 2014, 70-74. 


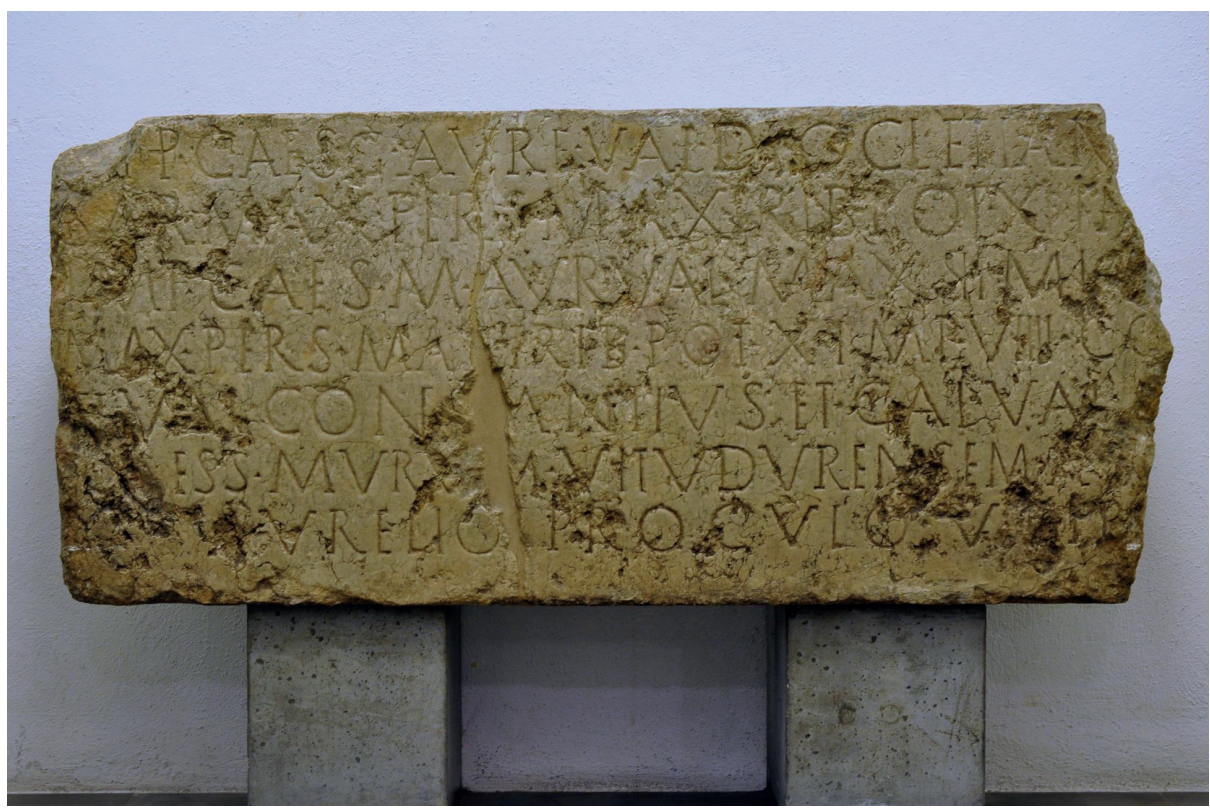

Abb. 2: Spätantike Bauinschrift aus dem Kastell in Oberwinterthur, die im Mittelalter und bis 1967 sichtbar in die Mauritiusrotunde am Konstanzer Münster eingemauert war (aus Maurer 1989, 72 Abb. (C) roland zh, https://de.wikipedia.org/wiki/Vitudurum\#/media/File:Vitudurum_-Oberwinterthur,_r\%C3\%B6mischer_Vicus_in_Winterthur_-_Gr\%C3\%BCndungsstein_des_Kastells_ im_Rathaus_2011-09-09_15-30-10_01.JPG [abgerufen am 27.01.2017]).

wöhnlicher Heiligkeit verehrt und entsprechend aufgesucht, wobei man an der Tafel die Hände zu reiben pflegte, um sich danach gleich über das Gesicht zu fahren. ${ }^{18}$

Diese Inschrift wurde neben der älteren, traditionsbildenden und der als Heiltum dienenden Funktion auch bestimmend für das Geschichtsbewusstsein der städtischen Einwohnerschaft. Im späten Mittelalter und der Frühen Neuzeit fand sie in Ursprungsgeschichten, städtischen und bischöflichen Chroniken sowie religionspolitischen Diskursen direkt oder indirekt breiten Niederschlag und diente dabei sowohl zur Betonung der weltlich-kaiserlichen wie der bischöflich-sakralen Tradition der Stadt. ${ }^{19}$

Somit lassen sich folglich mehrere Ebenen der Wahrnehmung und Deutung erkennen, nämlich erstens eine das Alter als Argumentation und Legitimationsstütze nutzende Ebene, zweitens eine volkstümliche, die Inschrift zum Heiltum uminterpretierende und drittens eine die städtischen Führungsschichten in ihrem Selbstbe-

18 Maurer 2014, 201; ausführlich auch Beck 2009, 130-137; vgl. die Edition des Textes Brunis bei Knittel 1979, 73-75.

19 Vgl. hierzu nun Eckhart 2016; Beck et al. 2009, 101-189. Zur Rezeptionsgeschichte der Inschrift während des Mittelalters vgl. auch Beck 2009, passim; Maurer 2014, 197-201. 
wusstsein bestärkende und für die Stadtgemeinde insgesamt identifikationsstiftende Ebene. ${ }^{20}$

Immer wieder sind während des Mittelalters weit zurückreichende imperiale Traditionen mit Hilfe noch vorhandener Inschriften konstruiert worden. Ein bislang wenig beachtetes Beispiel lässt sich für Baden-Baden belegen. ${ }^{21}$ Verschriftlicht wurde dieses - wie Theo Kölzer überzeugend aufzeigen konnte - im Kontext mehrerer Urkundenfälschungen, welche das im Unterelsass gelegene Reichskloster Weissenburg zu Beginn des 12. Jahrhunderts anfertigen ließ und die allesamt die Übertragung von Reichsbesitzungen zum Inhalt haben. ${ }^{22}$ Die hier interessierende Urkunde ${ }^{23}$ ist auf das Jahr 712 datiert, was sich aus dem in der Datumszeile genannten zweiten Regierungsjahr König Dagoberts und dem als Intervenienten genannten Weissenburger Abt Ratfried ergibt, welcher der Klostertradition zufolge von 695 bis 724 amtierte; gemeint war also Dagobert III.

Der Urkundentext führt aus, Dagobert habe ,auf Bitten des ehrwürdigen Abtes Ratfried [...] die jenseits des Rheins im Pagus Auciacinse gelegenen Thermen (balneas), welche die Kaiser Antoninus und Hadrian auf ihre Kosten erbauen ließen, dem Kloster des hl. Petrus zu Weissenburg übertragen [...]“. ${ }^{24}$ Die vorgebliche Schenkung betraf neben den Badeanlagen selbst noch weiteres Zubehör inklusive der zu den Bädern gehörigen Mark, deren Ausdehnung in dem Falsifikat beschrieben wird.

Durch die anschließende Grenzbeschreibung mit der Erwähnung des Flusses Murg wird ersichtlich, dass es sich um das heutige Baden-Baden handelt, das römische Aquae, ein Ort, der in der Antike zeitweilig als wichtiges Verwaltungszentrum der Provinz Germania Superior fungierte und aufgrund seiner warmen Quellen als Heilkurort aufgesucht wurde. ${ }^{25}$

Im weiteren Wortlaut der Grenzbeschreibung innerhalb der Urkunde werden die Thermen noch einmal weiter spezifiziert: de supra scriptis balneis, quae dicuntur Aquas Calidas, also Bäder, die auch als Warme Quellen bezeichnet werden. ${ }^{26}$ Die Fälschung benennt mit dieser Ortsbezeichnung eindeutig den antiken Bäderbezirk

20 Beck 2014, 350f.; vgl. zum Gegensatz zwischen bischöflicher und städtischer Geschichtsargumentation auch Eckhart 2016, 435-485; Eckhart 2009; Beck 2009, 121-123, 135-137.

21 Vgl. künftig die Publikation der Dissertation Beck 2015, 221-243 (Abgabefassung); bis dahin Beck 2014, 344-349 mit weiterführender Literatur.

22 Zur Begründung des Fälschungsvorwurfs und der Fälschungsserie siehe Kölzer 1998, $18 f$. ; vgl. auch Kölzer 1998/99, hier Bd. 2, 136-143.

23 MGH DD Merov., 402-405 Nr. 162.

24 Ideo cognoscat magnitudo seu utilitas vestra, quia nos ad suggestionem viri venerabilis Ratfridi abbatis de monasterio Wizunburgo balneas illas trans Rhenum in pago Auciacinse sitas, quas Antoninus et Adrianus quondam imperatores suo opere aedificaverunt, ad monasterium quod dicitur Wizenburg et est constructum in honore sancti Petri in pago Spirense visi fuimus concessisse cum omnibus et cum ipsa marca [...]. MGH DD Merov., 402-405 Nr. 162; Übersetzung nach Schwarzmaier 1988, 30.

25 Zur Geschichte der römischen Siedlung in Baden-Baden und den archäologischen Erkenntnissen über die dortigen Badeanlagen vgl. Schallmayer 1989; Mayer-Reppert/Rabold 2008.

26 MGH DD Merov., 402-405 Nr. 162. 
Baden-Badens, insbesondere die beiden dortigen monumentalen Thermenanlagen, deren mittlerweile wieder freigelegte Überreste noch heute zu besichtigen sind (Abb. 3). Die in der Urkunde solcherart festgehaltenen Bestimmungen zu den angeblich durch die Kaiser Antoninus und Hadrian errichteten Bädern waren aus Sicht des Klosters Weissenburg nur dann zielführend und verständlich, wenn man einen ansehnlichen Erhaltungszustand der Thermen noch zur Zeit der Fälschung im frühen 12. Jahrhundert voraussetzt, diese also vom Adressatenkreis des Fälschers noch zu identifizieren waren. ${ }^{27}$

Aus der schriftlichen Überlieferung des 10. und 11. Jahrhunderts ist die Vergabe von Reichsgut in Baden-Baden bezeugt. ${ }^{28}$ Teile hiervon werden etwa 1046 durch Heinrich III. dem Speyerer Domkapitel übertragen, in dessen Diözese Weissenburg lag. ${ }^{29}$ Bedeutsamer für die Motivation zur Erstellung der uns hier interessierenden Urkunde ist aber offenbar die Tatsache, dass im Zuge des staufisch-zähringischen Ausgleichs von 1098 neben den Entschädigungen für Berthold II. für seine Aufgabe der Ansprüche auf das Herzogtum Schwaben auch dessen Neffe Markgraf Hermann II. von königlicher Seite bedacht wurde und zwar höchstwahrscheinlich unter anderem mit umfangreichem Besitz in Baden-Baden - wonach sich dieser Familienzweig seit dem frühen 12. Jahrhundert fortan auch benannte. ${ }^{30}$ Die Übertragung von Reichsrechten in Baden-Baden an die selbstbewusst agierende und königsfähige Familie aber musste in der Abtei Weissenburg größte Sorgen um deren dortige Besitzungen und Rechte hervorrufen.

In diesem Zusammenhang sollte die Weissenburger Fälschung des frühen 12. Jahrhunderts für die klösterlichen Güter in Baden-Baden größtmögliche Autorität und vermeintliche Authentizität erzeugen. Die darin zu fassende Nennung der beiden antiken Herrscher, die während des Mittelalters - anders als vielerorts Julius

27 Beck 2014, 348f. Auch aus älteren Quellen ergibt sich unabhängig von diesem Zeugnis ein Wissen um die Reste des monumentalen Thermenensembles, wenn etwa im späten 10. Jahrhundert in der Vita des Augsburger Bischofs Udalrich ein Etappenort als ad balneos (Baden-Baden) Erwähnung findet; Gerhard von Augsburg 1986, 154f. Zu archäologischen Hinweisen auf eine lange Existenz der Ruinen vgl. Kaiser 1997, 127f. Sowohl die Baureste der „Kaiserbäder“ wie der „Soldatenbäder“ lagen zum Zeitpunkt ihrer Aufdeckung nicht unter tiefen Schuttschichten verborgen, sondern kamen nah unter der damaligen Bodenoberfläche zum Vorschein. Vgl. hierzu Rabold 2008, 13 Abb. 7 u. 8 sowie Mayer-Reppert 2008, 26 Abb. 26 u. 27.

28 MGH DD Germ. 2.2, 438 Nr. 39 (987, August 27); Baden-Baden als Actum-Ort: MGH DD Germ. 2.2, 563-565 Nr. 153 (994, August 28); früher bezogen auf Badenweiler. Zur Identifizierung mit BadenBaden vgl. Schwarzmaier 2005, hier 263 mit weiterführender Literatur. Zum Königshof Baden-Baden vgl. Maurer 2004, bes. $13 \mathrm{f}$.

29 MGH DD Germ. 5, 214-215 Nr. 172 (1046, September 9). Bestätigt durch Heinrich IV. 1101 vgl. MGH DD Germ. 6.2, 629-632 Nr. 466. Vgl. zu weiteren Nachweisen für Reichsgut Beck 2014, 348; künftig die Druckfassung der Dissertation Beck 2015, 225f. (Abgabefassung).

30 Schmid 1990, bes. 52ff.; Schmid 1992, 26; Schwarzmaier 2005, 259-263. 


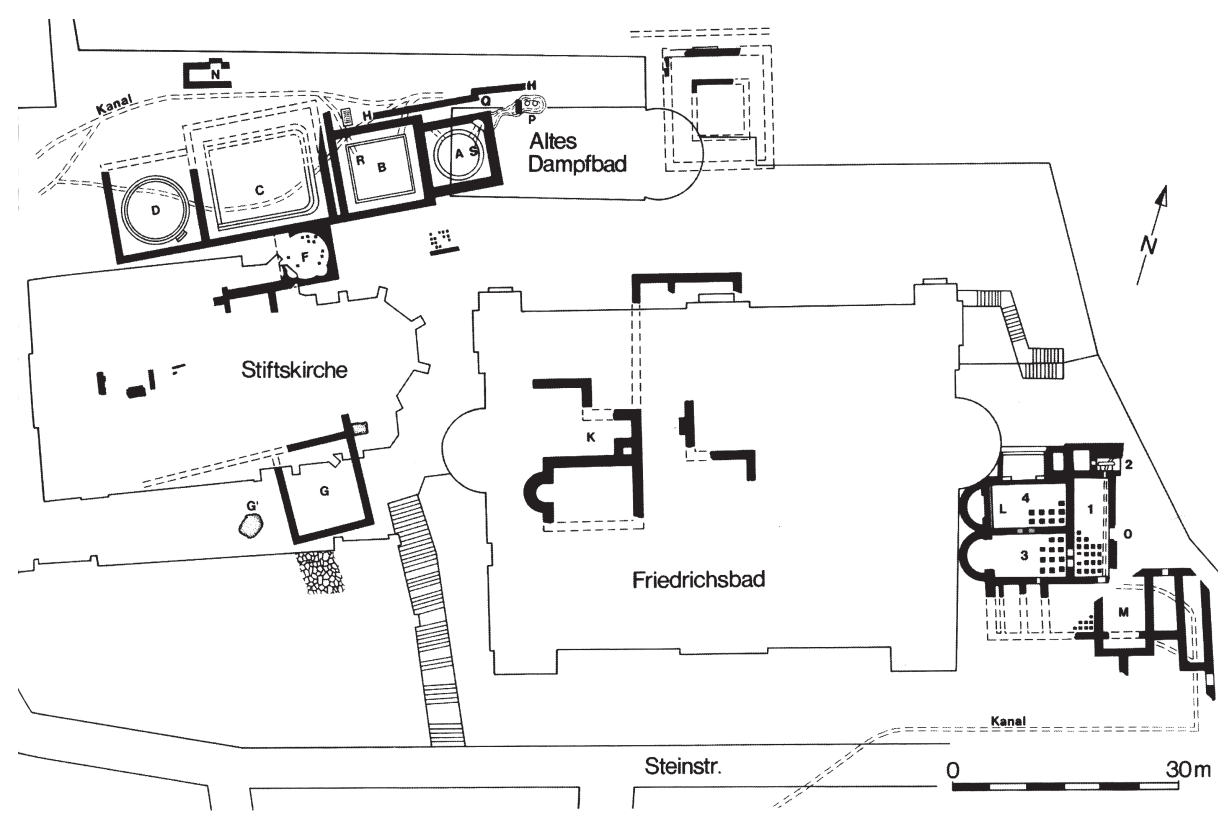

Abb. 3: Topographie des antiken Bäderbezirks von Baden-Baden mit späterer Überbauung (nach Mayer-Reppert/Rabold 2008, 23 Abb. 23).

Caesar $^{31}$ - nicht zu den stereotypen Gründerpersönlichkeiten zählten, lässt sich am ehesten auf im Mittelalter noch erhaltene (Bau-)Inschriften zurückführen. Hierfür kämen epigraphische Zeugnisse in Betracht, welche Hadrian nennen bzw. einen Kaiser, der den Bestandteil Antoninus im Titel führte, etwa Antoninus Pius, Marc Aurel oder Caracalla.

Tatsächlich sind antike Inschriften aus Baden-Baden in großer Zahl überliefert. ${ }^{32}$ Bis 1804 war etwa am Hauptportal der Stiftskirche eine Inschrift vermauert, die eine Widmung der res publica Aquensis an Caracalla nennt, wobei der Kaiser wie üblich als Marcus Aurelius Antoninus angesprochen wird (Abb. 4). ${ }^{33}$ Zuletzt wurde aufgrund der Titulatur des Kaisers Caracalla (Marcus Aurelius Severus Antoninus Pius), von dem mehrere Inschriften, darunter auch eine aus den Baden-Badener Thermen, vorliegen, davon ausgegangen, dass die Kaisernamen in der hochmittelalterlichen Fälschung aus zwei Inschriften, einer verlorenen auf Hadrian und einer 1848 in den Baden-Badener Thermen gefundenen auf Caracalla, entlehnt wurden. ${ }^{34}$

$31 \mathrm{Zu}$ Caesar als Heros in Ursprungsgeschichten und Literatur vgl. Clemens 2003, 342-356; Leeker 1986; zur Bedeutung Caesars für ein frühes „Nationalbewusstsein“ in Anno-Lied und Kaiserchronik auch Thomas 1992, 245-277 und zuletzt differenziert Mertens 2014, bes. 385-392.

32 Riedel 1975.

33 CIL XIII 6300; vgl. auch Filtzinger/Planck/Cämmerer 1986, 232f.; Rabold 2008, 9.

34 CIL XIII 6301 u. 6312. Die in zwei Teile zerbrochene und fragmentarische Bauinschrift nennt wiederum Marcus Aurelius Antoninus Pius, somit Caracalla, als Initiator und Finanzier einer Erweite- 


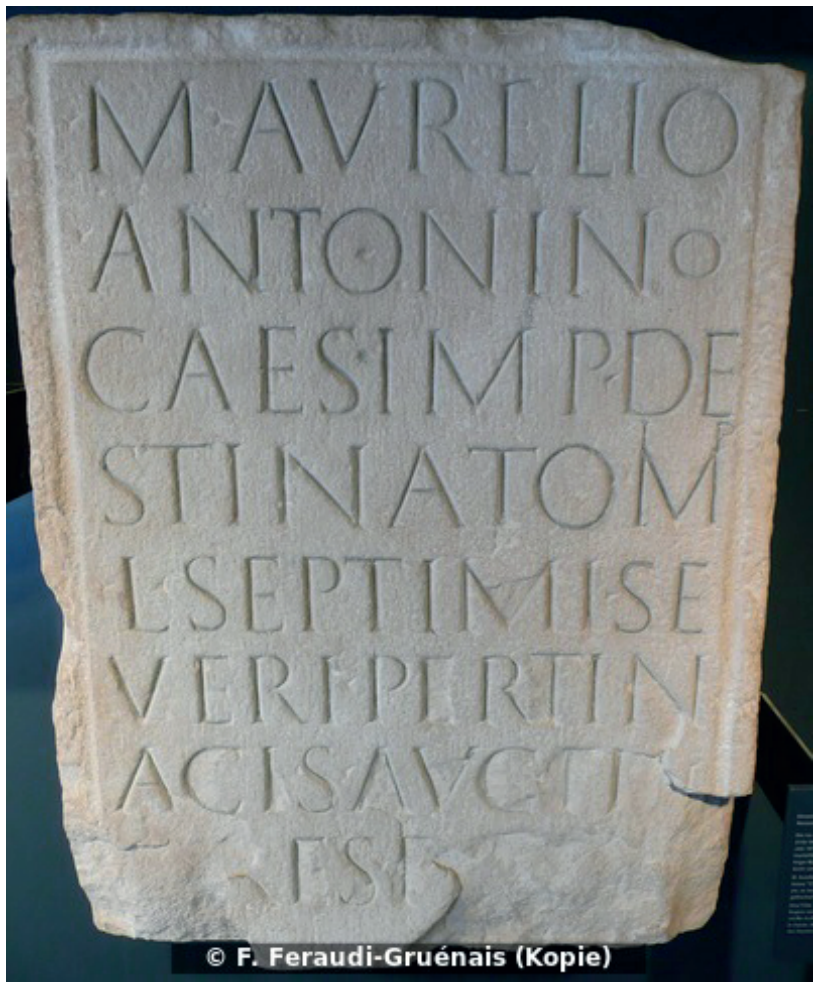

Abb. 4: Ehreninschrift für Caracalla aus Baden-Baden @ EDH F036047

Plausibler erscheint aber, als Vorlage eine heute verlorene Bauinschrift mit Nennung des Kaisers Antoninus Pius (138-161) anzunehmen, der sich seit 138 n. Chr. „T. Aelius Hadrianus Antoninus (Pius)“ nannte. ${ }^{35}$ Als Adoptivsohn des Hadrian hatte dessen Name Eingang in seine Titulatur gefunden. Träfe die Annahme zu, dann läge den in der auf 712 datierten Urkunde genannten Erbauern der Thermen von Baden-Baden lediglich ein epigraphisches Zeugnis zugrunde, wobei die dort lesbare Titulatur des Antoninus Pius auf zwei Herrscher (Antoninus und Hadrian) bezogen worden war.

Die Weissenburger Fälschung des frühen 12. Jahrhunderts entstand vor dem oben skizzierten Hintergrund mit der Intention, ein möglichst hohes Alter der ehemals aus Reichsbesitz stammenden klösterlichen Besitztitel in Baden-Baden zu kreieren. Diese schienen in Folge des staufisch-zähringischen Ausgleichs von 1098 durch den Eintritt Markgraf Hermanns II. in die Positionen des Reiches am Ort gefährdet. In diesem Kontext halfen die einer Inschrift entnommenen Namen zweier Kaiser und die

rung sowie der Wiederherstellung der Warmbäder. Der Inschrift zufolge ließ er das Bad mit Marmorplatten ausstatten. Zur Vermutung, diese Inschrift sei eine der Vorlagen des Fälschers gewesen, vgl. Beck 2014, 349.

35 Kienast 1990, 134. 
konstruierte Schenkung durch König Dagobert, klösterliche Rechtsposition zu stärken und die prestigeträchtige Herkunft des Besitzes aus Reichsgut zu untermauern. ${ }^{36}$

Ein letzter hier vorgestellter - aus heutiger Sicht abenteuerlich anmutender Versuch, aus einer Inschrift antik-imperiale Traditionen abzuleiten, führt uns schließlich nach Österreich. So berichtet die im letzten Drittel des 12. Jahrhunderts entstandene jüngere Passio Quirini vom Fund einer Inschrift in antiken Ruinen bei Iuvavum, also Salzburg, deren Text wie folgt gelautet haben soll:

Tempore Augusti Cesaris fuit Fabianus rex Romanorum, Juvavensium et totius Germaniae, post hunc Antoninus, post hunc Severus. ${ }^{37}$

Mit Hilfe dieses bereits auf den ersten Blick als zeitgenössische Konstruktion zu entlarvenden Quellenzeugnisses leitet der Autor einen Anspruch der Herzöge von Bayern auf das deutsche Königtum ab, denn auf den genannten Fabianus, der offenbar als Urbaier eingeführt wird, hätten sich angeblich Tassilo und Arnulf bei ihrem Kampf um die Königskrone berufen. Die auf der Basis einer antiken Inschrift argumentierende Geschichtskonstruktion ist im Zusammenhang mit den Auseinandersetzungen zwischen den bayerischen Welfen und den Staufern um die Vorherrschaft im Reich zu sehen, wobei für die Welfen Position bezogen wird. ${ }^{38}$ Zugleich wird das Alter Salzburgs, von dessen antiken Ruinen - tempore Romanorum constructa - wir seit dem ausgehenden 8. Jahrhundert aus den Gesta Hrodberti unterrichtet sind, ${ }^{39}$ in augusteische Zeit datiert.

Johann Weissensteiners scharfsinnigen Untersuchungen ist es $\mathrm{zu}$ verdanken, dass wir heute von einer verlorenen antiken Salzburger Inschrift ausgehen können, aus welcher der Verfasser der jüngeren Passio Quirini den wiedergegebenen Text konstruierte. Aus dieser Vorlage dürfte er die Kaisernamen und auch den des Fabianus entnommen haben. Ein L. Annius Fabianus war nämlich im Jahr 201 römischer Konsul. Er erscheint in dieser Funktion auch auf einer weiteren fragmentarischen Salzburger Inschrift. ${ }^{40}$ Seine Amtszeit fällt in die Regierung des Septimius Severus, der im Jahr 197 M. Aurelius Antoninus (den späteren Caracalla) zum Mitherrscher erhoben hatte, die beide in dem mittelalterlichen Text als Antoninus und Severus wiederzufinden sind. Folglich wird deutlich, dass der Geschichtskonstruktion des 12. Jahrhunderts eine Inschrift des Jahres 201 zugrunde lag, die womöglich auch jene in der Fälschung genannten Iuvanenses - etwa als Dedikanten - genannt haben könnte. ${ }^{41}$

36 Vgl. hierzu zuletzt Beck 2014, 349; künftig die Publikation der Dissertation Beck 2015, 242f. (Abgabefassung).

37 Weissensteiner 1983, 258.

38 Weissensteiner 1983, 200; Störmer 1988, 454f.

39 MGH SS rer. Merov. 6, 159f.; vgl. hierzu Clemens 2003, 260f.

40 Hainzmann/Schubert 1986, 55 Nr. 1090.

41 Weissensteiner 1983, 200-202. 
Soweit die wenigen hier kurz vorgestellten, im Wesentlichen hochmittelalterlichen Beispiele. Sie stehen stellvertretend für einen enormen Bestand an damals noch verfügbaren antiken Texten. Eine Chance, in der schriftlichen Überlieferung berücksichtigt zu werden, besaßen sie vor allem aber dann, wenn derartige Zeugnisse als Argumente in Geschichtskonstruktionen oder Traditionen einbezogen wurden. In Einzelfällen wurden Texte vollständig - wie in Deutz - oder teilweise - wie in BadenBaden, Waiblingen bzw. Salzburg - verstanden. In den überwiegenden Fällen waren die Rezipienten aber nur noch in der Lage, einzelne Herrschernamen zu lesen, die gleichwohl zur Begründung von Traditionen instrumentalisiert werden konnten.

\section{Literaturverzeichnis}

Althoff, Gerd (1988), „Genealogische und andere Fiktionen in mittelalterlicher Historiographie“, in: Fälschungen im Mittelalter (Tagung München 1986; MGH Schriften 33,1), Hannover, 417-441.

Andrikopoulou-Strack, Jeanne-Nora (1986), Grabbauten des 1. Jahrhunderts $n$. Chr. im Rheingebiet. Untersuchungen zu Chronologie und Typologie (Beihefte der Bonner Jahrbücher 43), Köln/ Bonn.

Beck, Erik et al. (Hgg.) (2009), „Altgläubige Bistumshistoriographie in einer evangelischen Stadt. Die Konstanzer Bistumschronik des Beatus Widmer von 1527: Untersuchung und Edition“, in: Zeitschrift für die Geschichte des Oberrheins 157, 101-189.

Beck, Erik (2009), „Argumentative Nutzung archäologischer Überreste im Kontext der Konstanzer Ursprungsgeschichte“, in: Erik Beck et al. (Hgg.), Altgläubige Bistumshistoriographie in einer evangelischen Stadt. Die Konstanzer Bistumschronik des Beatus Widmer von 1527: Untersuchung und Edition (Zeitschrift für die Geschichte des Oberrheins 157), 121-137.

Beck, Erik (2014), ,Deinde ad munimen Romani exercitus castella in circuitu munivit. Zur Wahrnehmung und Funktion römischer Überreste am Oberrhein“, in: Sebastian Brather et al. (Hgg.), Antike im Mittelalter. Fortleben - Nachwirken - Wahrnehmung (Archäologie und Geschichte. Freiburger Forschungen zum ersten Jahrtausend in Südwestdeutschland 21), Ostfildern, 329-354.

Beck, Erik (2015 i. Dr.), Mittelalterliche Wahrnehmung und Nutzung römerzeitlicher Überreste am südlichen Oberrhein unter besonderer Berücksichtigung der Burgen, Diss. Freiburg i. Br.

Berlioz, Jacques (1998), Catastrophes naturelles et calamités au Moyen Âge (Micrologus Library 1), Florenz.

Calabi Limentani, Ida (1970), „Sul non saper leggere le epigrafi classiche nei secoli XII e XIII. Sulla scoperta graduale delle abbreviazioni epigrafiche. A proposito di un libro recente“, in: Acme 23 (3), 253-282; zuletzt auch in: Calabi Limentani, Ida (Hg.) (2010), Scienza epigrafia. Contributi alla storia degli studi di epigrafia latina (Epigrafia e Antichità 28), Faenza, 11-42.

$\mathrm{CIL}=$ Corpus Inscriptionum Latinarum

Clemens, Lukas (2003), Tempore Romanorum constructa. Zur Nutzung und Wahrnehmung antiker Überreste nördlich der Alpen während des Mittelalters (Monographien zur Geschichte des Mittelalters 50), Stuttgart.

Clemens, Lukas (2009), „Zum Umgang mit Grabbauten der frühen und mittleren Kaiserzeit während der Spätantike und des Mittelalters nördlich der Alpen“, in: Dietrich Boschung (Hg.), Grabbauten des 2. und 3. Jahrhunderts in den gallischen und germanischen Provinzen (Tagung Köln 
2007; Schriften des Lehr- und Forschungszentrums für die antiken Kulturen des Mittelmeerraumes 7), Wiesbaden, 313-329.

Cüppers, Heinz/Binsfeld, Wolfgang (1972), „Eine zweiseitige beschriftete Grabplatte aus der St. Matthias-Basilika in Trier“, in: Trierer Zeitschrift 35, 135-140.

Die Deutschen Inschriften (2007), Bd. 70: Die Inschriften der Stadt Trier I (bis 1500), ges. u. bearb. von Rüdiger Fuchs, Wiesbaden.

Drack, Walter (1968/69), „Spätrömisches Kastell Vitudurum“, in: Bericht der Zürcher Denkmalpflege 6, 157-160.

Eckhart, Pia (2009), „Bischof gegen Rat. Die herrschaftspolitische Dimension der Bistumschronik im Kontext der 1520er Jahre“, in: Beck et al. (Hgg.), Altgläubige Bistumshistoriographie in einer evangelischen Stadt. Die Konstanzer Bistumschronik des Beatus Widmer von 1527: Untersuchung und Edition (Zeitschrift für die Geschichte des Oberrheins 157), 139-150.

Eckhart, Pia (2016), Ursprung und Gegenwart. Geschichtsschreibung in der Bischofsstadt und Werk des Konstanzer Notars Beatus Widmer (1475-ca. 1533) (Veröffentlichungen der Kommission für geschichtliche Landeskunde in Baden-Württemberg B 207), Stuttgart.

Erdmann, Wolfgang/Zettler, Alfons (1977), „Zur Archäologie des Konstanzer Münsterhügels“, in: Schriften des Vereins für Geschichte des Bodensees und seiner Umgebung 95, 19-134.

Esch, Arnold (2005), Wiederverwendung von Antike im Mittelalter. Die Sicht des Archäologen und die Sicht des Historikers (Hans-Lietzmann-Vorlesungen 7), Berlin/New York.

Filtzinger, Philipp/Planck, Dieter/Cämmerer, Bernhard (Hgg.) (1986), Die Römer in BadenWürttemberg, Stuttgart u. a.

Frézouls, Edmond (Hg.) (1988), Germanie Supérieure, Bd. 2.1: Besançon - Dijon - Langres Mandeure (Les Villes Antiques de la France), Straßburg.

Gabelmann, Hanns (1973), „Römische Grabmonumente mit Reiterkampfszenen. Mit einem epigraphischen Anhang von Géza Alföldi“, in: Bonner Jahrbücher 173, 132-200.

Gechter, Marianne (1989), „Das Kastell Deutz im Mittelalter“, in: Kölner Jahrbuch für Vor- und Frühgeschichte 22, 373-416.

Gechter, Marianne (1991), „Zur Überlieferung der Bauinschrift des Kastells Divitia (Deutz)“, in: Kölner Jahrbuch für Vor- und Frühgeschichte 24, 377-380.

Gerhard von Augsburg (1986), „Vita sancti Udalrici episcopi Augustani auctore Gerhardo“, übers. von Hatto Kallfelz, in: Hatto Kallfelz (Hg.), Lebensbeschreibungen einiger Bischöfe des 10.-12. Jahrhunderts (Ausgewählte Quellen zur deutschen Geschichte des Mittelalters. Freiherr vom Stein-Gedächtnisausgabe 22) Darmstadt, 37-167.

Grundmann, Herbert (1966), „Der Brand von Deutz 1128 in der Darstellung Abt Ruperts von Deutz. Interpretation und Text-Ausgabe“, in: Deutsches Archiv zur Erforschung des Mittelalters 22, 385-471.

Grünewald, Thomas (1989), „Ein epigraphisches Zeugnis zur Germanenpolitik Konstantins des Großen: Die Bauinschrift des Deutzer Kastells (CIL XIII 8502)“, in: Heinz E. Herzig u. Regula Frei-Stolba (Hgg.), Labor omnibus unus. Gerold Walser zum 70. Geburtstag dargebracht von Freunden, Kollegen und Schülern (Historia Einzelschriften 60), Stuttgart, 171-185.

Hainzmann, Manfred/Schubert, Peter (Hgg.) (1986), Inscriptionum lapidariarum Latinarum provinciae Norici usque ad annum MCMLXXXIV reperatarum Indices (ILLPRON Indices), Bd. 1: Catalogus, Berlin.

Hatt, Jean-Jacques (1951), La tombe gallo-romaine. Recherches sur les inscriptions et les monuments funéraires gallo-romains des trois premiers siècles de notre ère, Paris.

Heiligmann, Jörg (2014), „Römische Orte und ihre Weiternutzung. Die römischen Kastelle mit besonderer Berücksichtigung des Kastells Constantia - Konstanz", in: Sebastian Brather et al. (Hgg.), Antike im Mittelalter. Fortleben - Nachwirken - Wahrnehmung (Archäologie und 
Geschichte. Freiburger Forschungen zum ersten Jahrtausend in Südwestdeutschland 21), Ostfildern, 65-80.

Kaiser, Hartmut (1997), „Ausgrabungen an den römischen Badruinen in Baden-Baden“, in: Archäologische Ausgrabungen in Baden-Württemberg 1996, 124-131.

Kienast, Dietmar (1990), Römische Kaisertabelle. Grundzüge einer römischen Kaiserchronologie, Darmstadt.

Kiessling, Adolph (1863), Anecdota Basileensia I. Academisches Programm, Basel.

Knittel, Hermann (1979), Ex historia Constantiae. Lateinische Quellen zur Geschichte der Stadt Konstanz, Konstanz.

Kölzer, Theo (1998), „Kloster Weißenburg und Baden-Baden“, in: Borchardt, Karl/Bünz, Enno (Hgg.), Forschungen zur Reichs-, Papst- und Landesgeschichte. Peter Herde zum 65. Geburtstag von Freunden, Schülern und Kollegen dargebracht, Stuttgart, 15-24.

Kölzer, Theo (1998/99), Merowingerstudien, 2 Bde. (Monumenta Germaniae Historica. Studien und Texte 21 u. 26), Hannover.

Krause, Rüdiger (1981), Von der Steinzeit bis zum frühen Mittelalter. Archäologische Bodenfunde im Stadtgebiet Waiblingen (Veröffentlichungen des Archivs der Stadt Waiblingen 2), Waiblingen.

Kremer, Gabrielle (2009), Das frühkaiserzeitliche Mausoleum von Bartringen (Luxemburg) mit einem Beitrag von Jean Krier (Dossiers d'Archéologie du Musée National d'Histoire et d'Art 12), Luxemburg.

Laurentius Surius (1571), De probatis Sanctorum historiis, Bd. 2, Köln.

Le Bohec, Yann (1991), „Un document connu et méconnu, le ,Testament du Lingon““, in: Yann Le Bohec (Hg.), Le Testament du Lingon (Tagung Lyon 1990; Collection du Centre d’Études Romaines et Gallo-romaines n. s. 9), Lyon, 9-15.

Le Glay, Marcel (1991), „Notes sur le ,Testament du Lingon““, in: Yann Le Bohec (Hg.), Le Testament du Lingon (Tagung Lyon 1990; Collection du Centre d’Études Romaines et Gallo-romaines n. s. 9), Lyon, 57-61.

Leeker, Joachim (1986), Die Darstellung Cäsars in den romanischen Literaturen des Mittelalters (Analecta Romanica 50), Frankfurt a. M.

Lorenz, Sönke (2000), Waiblingen - Ort der Könige und Kaiser (Gemeinde im Wandel 13), Waiblingen.

Lot, Ferdinant (1922), „Itinéraires du XIIle siècle. I. De Valenciennes à Avignon. II. De Lyon à Orléans et à Paris suivis d'un relevé de quelques curiosités qu'on rencontre sur ses routes“, in: Bulletin Philologiques et Historique du Comité des Travaux Historiques et Scientifiques, 217-222.

Maurer, Helmut (1973), Konstanz als ottonischer Bischofssitz. Zum Selbstverständnis geistlichen Fürstentums im 10. Jahrhundert (Veröffentlichungen des Max-Planck-Instituts für Geschichte 39; Studien zur Germania Sacra 12), Göttingen.

Maurer, Helmut (1989), Konstanz im Mittelalter (Geschichte der Stadt Konstanz 1), Konstanz.

Maurer, Helmut (2004), „Baden-Baden (B)“, in: Helmut Maurer (Bearb.), Die deutschen Königspfalzen, Bd. 3: Baden-Württemberg, Teilband 1: Adelberg - Reichenau, Göttingen, 8-17.

Maurer, Helmut (2014), „Spätrömische Kastellorte und die Anfänge des Bistums Konstanz“, in: Sebastian Brather et al. (Hgg.), Antike im Mittelalter. Fortleben - Nachwirken - Wahrnehmung (Archäologie und Geschichte. Freiburger Forschungen zum ersten Jahrtausend in Südwestdeutschland 21), Ostfildern, 195-213.

Mayer-Reppert, Petra/Rabold, Britta (Hgg.) (2008), Die römischen,Soldatenbäder ' in Baden-Baden (Aquae Aureliae) (Führer zu archäologischen Denkmälern in Baden-Württemberg 25), Stuttgart. Mayer-Reppert, Petra (2008), „Die römischen Badeanlagen“, in: Petra Mayer-Reppert u. Britta Rabold (Hgg.), Die römischen, Soldatenbäder in Baden-Baden (Aquae Aureliae) (Führer zu archäologischen Denkmälern in Baden-Württemberg 25), Stuttgart, 23-57. 
Mertens, Dieter (2014), „Caesar, Arminius und die Deutschen. Meistererzählungen und Aitiologien“, in: Sebastian Brather et al. (Hgg.), Antike im Mittelalter. Fortleben - Nachwirken - Wahrnehmung (Archäologie und Geschichte. Freiburger Forschungen zum ersten Jahrtausend in Südwestdeutschland 21), Ostfildern, 383-441.

MGH DD Germ. = Monumenta Germaniae Historica. Diplomata regum et imperatorum Germaniae (Die Urkunden der deutschen Könige und Kaiser)

MGH DD Merov. = Monumenta Germaniae Historica. Diplomata regum Francorum e stirpe Merovingica (Die Urkunden der Merowinger)

MGH SS = Monumenta Germaniae Historica. Scriptores (in Folio)

MGH SS rer. Merov. = Monumenta Germaniae Historica. Scriptores rerum Merovingicarum

Rabold, Britta (2008), „Baden-Baden - Topografie und Kenntnisstand“, in: Petra Mayer-Reppert u. Britta Rabold (Hgg.), Die römischen ,Soldatenbäder‘ in Baden-Baden (Aquae Aureliae) (Führer zu archäologischen Denkmälern in Baden-Württemberg 25), Stuttgart, 9-22.

Reuter, Timothy (1992), „Past, Present and No Future in the Twelfth-Century Regnum Teutonicum“, in: Paul Magdalino (Hg.), The Percepton of the Past in Twelfth-Century Europe, London, 15-36.

Riedel, Matthias (1975), Civitas Aurelia Aquensis. Die Geschichte des römischen Baden-Baden anhand der Kleinfunde und der Inschriften, Diss. masch.-schriftl. Freiburg i. Br.

Ruelens, Charles (1890), „Comment jadis on se rendait à Rome“, in: Bulletin de la Société Royale Belge de Géographie 14, 301-330.

Schallmayer, Egon (1989), Aquae - das römische Baden-Baden (Führer zu archäologischen Denkmälern in Baden-Württemberg 11), Stuttgart.

Schmid, Karl (1976), „De regia stirpe Waiblingensium. Bemerkungen zum Selbstverständnis der Staufer“, in: Zeitschrift für die Geschichte des Oberrheins 124, 63-73.

Schmid, Karl (1990), „Zürich und der staufisch-zähringische Ausgleich 1098“, in: Karl Schmid (Hg.), Die Zähringer, Bd. 3: Schweizer Vorträge und Forschungen, Sigmaringen, 49-79.

Schmid, Karl (1992), „Baden-Baden und die Anfänge der Markgrafen von Baden“, in: Zeitschrift für die Geschichte des Oberrheins 140, 1-37.

Schwarzmaier, Hansmartin (1988), Baden-Baden im frühen Mittelalter. Die älteste schriftliche Überlieferung aus den Klöstern Weissenburg und Selz, Baden-Baden.

Schwarzmaier, Hansmartin (2005), „Selz im Machtbereich der Staufer und der Markgrafen von Baden im Hochmittelalter“, in: Franz Staab u. Thorsten Unger (Hgg.), Kaiserin Adelheid und ihre Klostergründung in Selz (Tagung Landau/Selz 1999; Veröffentlichungen der Pfälzischen Gesellschaft zur Förderung der Wissenschaften 99), Speyer, 259-277.

Störmer, Wilhelm (1988), „Beobachtungen zu Aussagen und Intentionen der bayerischen Stammes,Sage“ des 11./12. Jahrhunderts. Fiktionen - Sage - ,Geschichtsklitterung،“, in: Fälschungen im Mittelalter (Tagung München 1986; MGH Schriften 33,1), Hannover, 451-470.

Thomas, Heinz (1992), „Julius Caesar und die Deutschen. Zu Ursprung und Gehalt eines deutschen Geschichtsbewußtseins in der Zeit Gregors VII. und Heinrichs IV“, in: Stefan Weinfurter (Hg.) unter Mitarbeit von Hubertus Seiler, Gesellschaftlicher und ideengeschichtlicher Wandel im Reich der Salier (Die Salier und das Reich 3), Sigmaringen, 245-277.

Weissensteiner, Johann (1983), Tegernsee, die Bayern und Österreich. Studien zu Tegernseer Geschichtsquellen und der bayerischen Stammessage. Mit einer Edition der Passio secunda S. Quirini (Archiv für österreichische Geschichte 133), Wien. 
